\title{
Flatness of heavy chain systems
}

\author{
Nicolas Petit ${ }^{1}$
}

Abstract

This paper gives an overview of the results of [11]. Furthermore it contains some previously unpublished material concerning the homogeneous chain carrying a load, see equation (13). In [11] the flatness [3, 4] of heavy chain systems, i.e. trolleys carrying a fixed length heavy chain that may carry a load, is addressed in the partial derivatives equations framework. We parameterize the system trajectories by the trajectories of its free end and solve the motion planning problem, namely steering from one state to another state. When considered as a finite set of small pendulums these systems were shown to be flat in [10]. Our study is an extension to the infinite dimensional case.

Under small angle approximations, these heavy chain systems are described by a 1D partial differential wave equation. Dealing with this infinite dimensional description, we show how to get the explicit parameterization of the chain trajectory using (distributed and punctual) advances and delays of its free end.

\section{Introduction}

The notion of flatness $[3,4]$ has proven to be relevant in many problems where motion planning problems have been solved $[9,5]$. The existence of a flat output is the key to explicit formulas that can be implemented as open-loop controllers.

The heavy chain systems under consideration in this paper are defined by a trolley carrying a fixed length heavy chain to which a load may be attached. The dynamics are studied in a fixed vertical plane. When approximated as a finite set of small pendulums, such heavy chain systems were shown to be flat (see [10]). Their trajectories can be explicitly parameterized by the trajectories of their free ends. These parameterizations involve numerous derivatives, (twice as many as the number of pendulums). When this number goes to infinity, the derivative order goes to infinity as well, yielding series expansions. This makes these relations

\footnotetext{
${ }^{1}$ Centre Automatique et Systèmes, École Nationale Supérieure des Mines de Paris, 60, bd. Saint-Michel, 75272 Paris Cedex 06, France nicolas.petit@ensmp.fr

${ }^{2}$ Centre Automatique et Systèmes, École Nationale Supérieure des Mines de Paris, 60, bd. Saint-Michel, 75272 Paris Cedex 06, France pierre.rouchon@ensmp.fr
}

\author{
Pierre Rouchon ${ }^{2}$
}

difficult to handle and to use in practice.

In order to overcome these difficulties, we consider infinite dimensional descriptions of heavy chains systems. Around the stable vertical steady-state and under the small angle assumption, the dynamics are described by second order ordinary differential equations (dynamics of the load at position $y(t))$ coupled with 1D wave equations (dynamics of the chain $X(x, t)$ ) where waves speed depends on $x$, the spatial variable along the chain length.

This combined ordinary and partial differential equation description turns out to be a significant shortcut to an explicit motion planning formula. Instead of an infinite number of derivatives, the explicit parameterization of the trajectories involves a small number of both distributed and punctual advances and delays. The controllability of such hybrid systems could be analyzed via Hilbert's uniqueness method [7, 8], as done in [6]. The work presented here is also a constructive proof of the controllability of these systems in the sense that it provides the open-loop control for steering the system from any given state to any other state. In a real application it should be used as a feed-forward term complemented by a closed-loop controller using the energy method as proposed in [2].

In the case of a single homogeneous heavy chain as depicted in figure 1 (see section 2 for details), our explicit parameterization shows that the general solution of

$$
\frac{\partial}{\partial x}\left(g x \frac{\partial X}{\partial x}\right)-\frac{\partial^{2} X}{\partial t^{2}}=0
$$

is given by the following integral

$$
X(x, t)=\frac{1}{2 \pi} \int_{-\pi}^{\pi} y(t+2 \sqrt{x / g} \sin \theta) d \theta
$$

where $t \mapsto y(t)$ is any smooth enough time function: $X(0, t)=y(t)$ corresponds then to the free end position; the control $u(t)=X(L, t)$ is the trolley position.

For the general cases, we have shown in [11] that relationship similar to (1) exist. They are expressed by equations (8) and (10) included here for convenience. The structure is similar but the moving averages involve kernels depending on the mass distribution. More precisely, given any mass distribution along the chain and any punctual mass at $x=0$, we prove that there is a one to one correspondence between the trajectory 

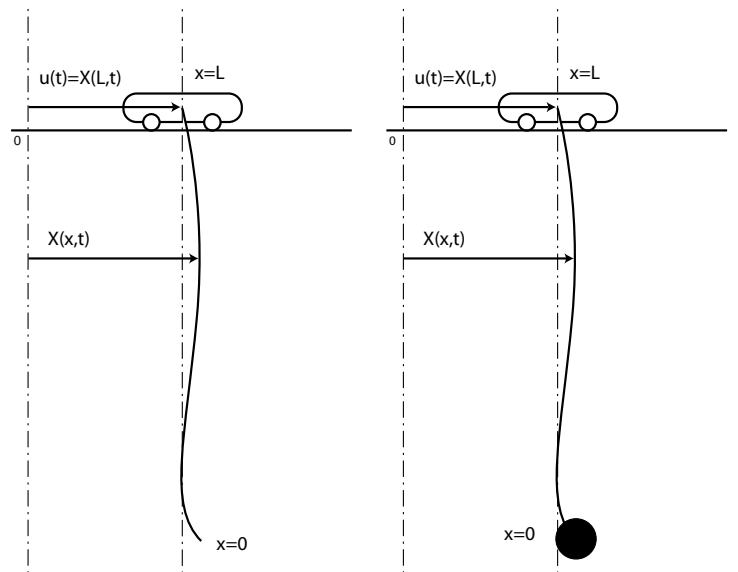

Figure 1: The homogeneous chain without or with any load.

of the load $t \mapsto y(t)=X(0, t)$ and the trajectory of the whole system (namely the cable and the trolley): $t \mapsto X(x, t)$ and $t \mapsto u(t)=X(L, t)$. This correspondence yields the explicit parameterization of the trajectories: $X(x, \cdot)=\mathcal{A}_{x} y$ where $\left\{\mathcal{A}_{x}\right\}$ is a set of operators including time derivations, advances and delays. In other words, $(x, t) \mapsto\left(\mathcal{A}_{x} y\right)(t)$ satisfies the system equations for any smooth function $t \mapsto y(t)$. For each $x$, the operator $\mathcal{A}_{x}$ admits compact support. Thus it is possible to steer the system from any initial point to any other point in finite time.

This parameterization results from symbolic computations. Replacing the time derivative by the Laplace variable $s$ yields a second order differential equation in $x$ with $s$ as a parameter. For each $x$, its fundamental solution $A_{x}$ is an entire function of $s$ of exponential type. Furthermore, for each $x$ we show, thanks to the Liouville transformation, that $s \mapsto A_{x}(s)$ satisfies the assumptions of the Paley-Wiener theorem, modulo explicitly computable exponentials of $s$.

We recall here the results for this general case and refer to [11] for proofs and details. Furthermore we give in the last section of this paper some previously unpublished material: analytical solutions for the homogeneous cable carrying a load which is a case of engineering interest.

\section{The homogeneous chain without any load}

Consider a heavy chain in stable position as depicted in figure 1. Under the small angle approximation it is ruled by the following dynamics

$$
\left\{\begin{array}{c}
\frac{\partial}{\partial x}\left(g x \frac{\partial X}{\partial x}\right)-\frac{\partial^{2} X}{\partial t^{2}}=0 \\
X(L, t)=u(t) .
\end{array}\right.
$$

where $x \in[0, L], t \in \mathbb{R}, X(x, t)-X(L, t)$ is the deviation profile, $g$ is gravitational acceleration and the control $u$ is the trolley position.

Thanks to the classical mapping $y=2 \sqrt{\frac{x}{g}}$, we get

$$
y \frac{\partial^{2} X}{\partial y^{2}}(y, t)+\frac{\partial X}{\partial y}(y, t)-y \frac{\partial^{2} X}{\partial t^{2}}(y, t)=0 .
$$

Use Laplace transform of $X$ with respect to the variable $t$ (denoted by $\hat{X}$ and with zero initial conditions i.e. $X(., 0)=0$ and $\left.\frac{\partial X}{\partial t}(., 0)=0\right)$ to get

$$
y \frac{\partial^{2} \hat{X}}{\partial y^{2}}(y, s)+\frac{\partial \hat{X}}{\partial y}(y, s)-y s^{2} \hat{X}(y, s)=0 .
$$

Less classically the mapping $z=\imath s y$ gives

$$
z \frac{\partial^{2} \hat{X}}{\partial z^{2}}(z, s)+\frac{\partial \hat{X}}{\partial z}(z, s)+z \hat{X}(z, s)=0 .
$$

This is a Bessel equation. Its solution writes in terms of $J_{0}$ and $Y_{0}$ the zero-order Bessel functions. Using the inverse mapping $z=2 \imath s \sqrt{\frac{x}{g}}$, we get

$$
\hat{X}(x, s)=A J_{0}(2 \imath s \sqrt{x / g})+B Y_{0}(2 \imath s \sqrt{x / g}) .
$$

Since we are looking for a bounded solution at $x=0$ we have $B=0$. Then

$$
\hat{X}(x, s)=J_{0}(2 \imath s \sqrt{x / g}) \hat{X}(0, s) .
$$

where we can recognize the Clifford function $\mathcal{C}_{1}$ (see $[1$, p 358]). Using Poisson's integral representation of $J_{0}$ $[1$, formula 9.1 .18$]$

$$
J_{0}(z)=\frac{1}{2 \pi} \int_{-\pi}^{\pi} \exp (\imath z \sin \theta) d \theta,
$$

we have

$$
J_{0}(2 \imath s \sqrt{x / g})=\frac{1}{2 \pi} \int_{-\pi}^{\pi} \exp (2 s \sqrt{x / g} \sin \theta) d \theta .
$$

In terms of Laplace transforms, this last expression is a combination of delay operators. Turning (4) back into the time-domain we get

$$
X(x, t)=\frac{1}{2 \pi} \int_{-\pi}^{\pi} y(t+2 \sqrt{x / g} \sin \theta) d \theta,
$$

with $y(t)=X(0, t)$.

Relation (5) means that there is a one to one correspondence between the (smooth) solutions of (2) and 
the (smooth) functions $t \mapsto y(t)$. For each solution of $(2)$, set $y(t)=X(0, t)$. For each function $t \mapsto y(t)$, set $X$ by $(5)$ and $u$ as

$$
u(t)=\frac{1}{2 \pi} \int_{-\pi}^{\pi} y(t+2 \sqrt{L / g} \sin \theta) d \theta
$$

to obtain a solution of (2).

Finding $t \mapsto u(t)$ steering the system from the steadystate $X \equiv 0$ at $t=0$ to the other one $X \equiv D$ at $t=T$ becomes obvious. Our analysis shows that $T$ must be larger than $2 \Delta$ where $\Delta=2 \sqrt{L / g}$ is the travelling time of a wave between $x=L$ and $x=0$. It just consists in finding $t \mapsto y(t)$ that is equal to 0 for $t \leqslant \Delta$ and to $D$ for $t>T-\Delta$ and in computing $u$ via (6).

Figure 2 illustrates computations based on (5) with

$y(t)=\left\{\begin{array}{l}0 \text { if } t<\Delta \\ \frac{3 L}{2}\left(\frac{t-\Delta}{T-2 \Delta}\right)^{2}\left(3-2\left(\frac{t-\Delta}{T-2 \Delta}\right)\right) \text { if } \Delta \leqslant t \leqslant T-\Delta \\ \frac{3 L}{2} \text { if } t>T-\Delta\end{array}\right.$ where the chosen transfer time $T$ equals $4 \Delta$. For $t \leqslant 0$ the chain is vertical at position 0 . For $t \geqslant T$ the chain is vertical at position $D=3 L / 2$.

Plots of figure 3 show the control $[0, T] \ni t \mapsto u(t)$ required for such motion. Notice that the support of $\dot{u}$ is $[0, T]$ while the support of $\dot{y}$ is $[\Delta, T-\Delta]$. To be consistent with the small angle approximation, the horizontal acceleration of the end point $\ddot{y}$ must be much smaller than $g$. In our computations the maximum of $|\ddot{y}|$ is chosen rather large, $9 g / 16$. This is just for tutorial reasons. In practice, a reasonable transition time is $T=5 \Delta$ yielding $|\ddot{y}| \leqslant g / 4$.

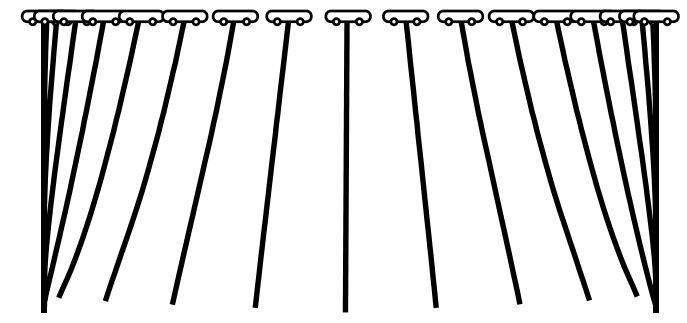

Figure 2: Steering from 0 to $3 L / 2$ in finite time $T=4 \Delta$. Regularly time-spaced positions of the heavy chain system are represented. The Matlab simulation code can be obtained from the second author via email.

\section{The inhomogeneous (i.e. variable section) chain without any load}

Formulas (5) can be extended to an heavy chain with variable section and carrying no load (see figure 4).

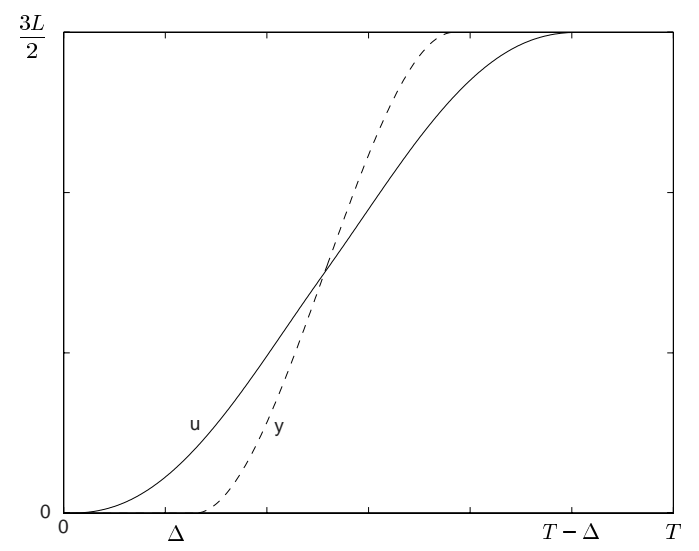

Figure 3: The steering control, trolley position $u$, and the "flat output", the free end $y$.
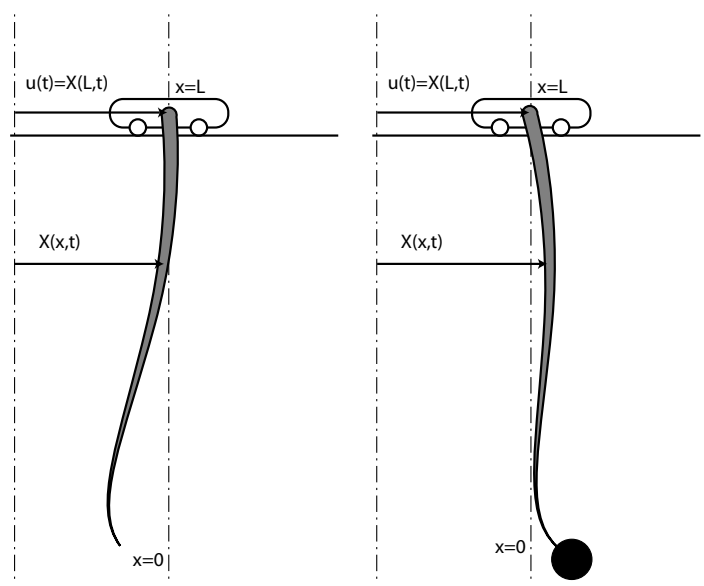

Figure 4: The inhomogeneous chain without or with load.

Such an extension deserves special consideration because of the singularity of the partial differential system at $x=0$.

Such a system is governed by the following equations

$$
\left\{\begin{array}{c}
\frac{\partial}{\partial x}\left(\tau(x) \frac{\partial X}{\partial x}\right)-\frac{\tau^{\prime}(x)}{g} \frac{\partial^{2} X}{\partial t^{2}}=0 \\
X(L, t)=u(t)
\end{array}\right.
$$

where $x \in[0, L], t \in \mathbb{R}$ and $u$ is the control. The tension of the chain is $\tau(x)$ with $\tau(0)=0$ and $\tau(x)=$ $g x+\mathcal{O}\left(x^{2}\right)$, while $\tau^{\prime}(x) / g>0$ is the mass distribution along the chain. Furthermore, we assume that there exist $a>0$ such that $\tau(x) \geqslant a x \geqslant 0$.

Theorem 1 Consider (7) with $[O, L] \ni x \mapsto \tau(x) a$ smooth increasing function with $\tau(0)=0$ and $\tau^{\prime}>$ 0 . There is a one to one correspondence between the solutions $[0, L] \times \mathbb{R} \ni(x, t) \mapsto(X(x, t), u(t))$ that are $C^{3}$ 


$$
\begin{aligned}
X(x, t)= & \frac{L^{1 / 4} \sqrt{g}}{2 \pi^{3 / 2}\left(\tau(x) \tau^{\prime}(x)\right)^{1 / 4}} \sqrt{G(2 \sqrt{\tau(x) / g})} \int_{-\pi}^{\pi} y(t+K G(2 \sqrt{\tau(x) / g}) \sin \theta) d \theta \\
& +\frac{1}{\left(\tau(x) \tau^{\prime}(x) / g\right)^{1 / 4}} \int_{-2 \sqrt{\frac{\tau(x)}{a g}}}^{2 \sqrt{\frac{\tau(x)}{a g}}} \mathcal{K}(G(2 \sqrt{\tau(x) / g}), \xi) \dot{y}(t+\xi) d \xi \\
u(t)= & X(L, t) \text { with } y(t)=X(0, t)
\end{aligned}
$$

in t and the $C^{3}$ functions $\mathbb{R} \ni t \mapsto y(t)$ via formulas (8) where the constant $K$ and the functions $G$ and $\mathcal{K}$ are uniquely defined by the function $\tau$.

The proof of this result is given in [11]. It is organized as follows

1. A simple time-scaling simplifies the system. We shift from $\mathrm{X}$ to $\mathrm{Y}$.

2. Symbolic computations where time derivatives are replaced by the Laplace variable $s$ are performed.

3. The solution $Y(x, s)$ is factorized as $Y(x, s)=$ $Y(0, s) A(x, s)$. A partial differential system is derived for $A(x, s)$.

4. A Liouville transformation is performed.

5. In these new coordinates the preceding transformed equation is compared to an equation that we have already solved in section 2, namely the equation of a single homogeneous chain. We denote by $D(x, s)$ the difference between these two solutions.

6. $D(x, s)$ is proven to be an entire function of $s$ and of exponential type.

7. A careful study of the Volterra equation satisfied by $D(x, s)$ shows that, for each $x$, the restriction to $D(x, s) / s$ to the imaginary axis is in $L^{2}$.

8. Thanks to the Paley-Wiener theorem, we prove that, for each $x, D(x, s) / s$ can be represented as a compact sum (discrete and continuous) of exponentials in $s$.

9. Gathering all the terms of $A(x, s)$ we get an expression involving the Bessel function $J_{0}$ (the solution for an homogeneous chain) and exponentials in $s$ multiplied by $s$. This gives equations (8).

\section{The inhomogeneous chain with punctual load}

The system of figure 4 consists of an heavy chain with a variable section carrying a punctual load $m$. Small deviations $X(x, t)-u(t)$ from the vertical position are described by the following partial differential system

$$
\left\{\begin{aligned}
\frac{\partial}{\partial x}\left(\tau(x) \frac{\partial X}{\partial x}\right) & -\frac{\tau^{\prime}(x)}{g} \frac{\partial^{2} X}{\partial t^{2}}=0 \\
\frac{\partial^{2} X}{\partial t^{2}}(0, t) & =g \frac{\partial X}{\partial x}(0, t) \\
X(L, t) & =u(t)
\end{aligned}\right.
$$

where $u$ is the control. The tension in the chain writes $\tau(x): \quad \tau(0)=m g$ and $\tau^{\prime}(x) / g>0$ is the mass distribution along the chain.

Theorem 2 Consider (11) with $[0, L] \ni x \mapsto \tau(x) a$ smooth increasing function with $\tau(0)=m$. There is a one to one correspondence between the solutions $[0, L] \times$ $\mathbb{R} \ni(x, t) \mapsto(X(x, t), u(t))$ that are $C^{3}$ in $t$ and the $C^{3}$ functions $\mathbb{R} \ni t \mapsto y(t)$ via the formulas (10)

where $B(x, \xi)$ a smooth function of $x$ and $\xi$ uniquely defined by the function $\tau$.

Correspondence (10) defines a family of linear operators $\mathcal{A}_{x}$ with compact support such that, for any $C^{3}$ time function, $X(x, t)=\left.\mathcal{A}_{x} y\right|_{t}$ is automatically solution of (11) with $u(t)=X(L, t)$ and $X(0, t)=y(t)$.

The proof of this result is given in [11]. It is different from the case without any load. The key issue is that one has to deal with the extra boundary condition $\frac{\partial^{2} X}{\partial t^{2}}(0, t)=g \frac{\partial X}{\partial x}(0, t)$. To overcome this, one has to use Volterra expansions before the Paley-Wiener theorem.

\section{The homogeneous chain with punctual load}

In the particular case of an homogeneous mass disstribution we are able to give explicit formulas for the motion planning problem using Bessel functions. Consider a trolley carrying an homogeneous chain and a punctual load

$$
\left\{\begin{aligned}
\frac{\partial}{\partial x}\left(g(x+a) \frac{\partial X}{\partial x}\right) & -\frac{\partial^{2} X}{\partial t^{2}}=0 \\
\frac{\partial^{2} X}{\partial t^{2}}(0, t) & =g \frac{\partial X}{\partial x}(0, t) \\
X(L, t) & =u(t)
\end{aligned}\right.
$$




$$
\left\{\begin{array}{c}
X(x, t)=\phi(x)[y(t+\theta(x))+y(t-\theta(x))]+\psi(x)[\dot{y}(t+\theta(x))-\dot{y}(t-\theta(x))] \\
+\int_{0}^{x} \mathcal{B}(x, \xi)[y(t+\theta(\xi))+y(t-\theta(\xi))] d \xi \\
u(t)=X(L, t)
\end{array}\right.
$$

with

$$
\begin{aligned}
y(t) & =X(0, t), \quad \theta(x)=\int_{0}^{x} \sqrt{\frac{\tau^{\prime}}{g \tau}}, \quad \psi(x)=\left(\frac{\tau(0) \tau^{\prime}(0)}{\tau(x) \tau^{\prime}(x)}\right)^{\frac{1}{4}} \frac{1}{2} \sqrt{\frac{\tau(0)}{g \tau^{\prime}(0)}} \\
\phi(x) & =\left(\frac{\tau(0) \tau^{\prime}(0)}{\tau(x) \tau^{\prime}(x)}\right)^{\frac{1}{4}} \ldots \\
& \times\left[1+\frac{1}{8} \sqrt{\frac{\tau(0)}{\tau^{\prime}(0)}}\left(\left(\sqrt{\frac{\tau^{\prime}}{\tau}}+\frac{\tau^{\prime \prime}}{\tau^{\prime}} \sqrt{\frac{\tau}{\tau^{\prime}}}\right)(x)-\left(\sqrt{\frac{\tau^{\prime}}{\tau}}+\frac{\tau^{\prime \prime}}{\tau^{\prime}} \sqrt{\frac{\tau}{\tau^{\prime}}}\right)(0)+\frac{1}{4} \int_{0}^{x}\left(\sqrt{\frac{\tau^{\prime}}{\tau}}+\frac{\tau^{\prime \prime}}{\tau^{\prime}} \sqrt{\frac{\tau}{\tau^{\prime}}}\right)^{2} \sqrt{\frac{\tau^{\prime}}{\tau}}\right)\right]
\end{aligned}
$$

where $u$ is the control. The homogeneous mass distribution is normalized to 1 by unit of length and so the coefficient $a$ stands for the mass of the load.

As in section 2, we use the mapping $z=2 \imath s \sqrt{\frac{x+a}{g}}$ after a Laplace transform to get as before

$$
\hat{X}(x, s)=A J_{0}\left(2 \imath s \sqrt{\frac{x+a}{g}}\right)+B Y_{0}\left(2 \imath s \sqrt{\frac{x+a}{g}}\right) .
$$

This time it is not possible to conclude that $B=0$ when using the boundedness of the solution as $x$ goes to 0 . This is the case only for $a=0$, see section 2 .

Anyway, we have

$$
\begin{aligned}
& \frac{\partial \hat{X}}{\partial x}(x, s)= \\
& -\frac{\imath s}{\sqrt{g(x+a)}}\left(A J_{1}\left(2 \imath s \sqrt{\frac{x+a}{g}}\right)+B Y_{1}\left(2 \imath s \sqrt{\frac{x+a}{g}}\right)\right) .
\end{aligned}
$$

The boundary condition $s^{2} \hat{X}(0, s)=g \frac{\partial \hat{X}}{\partial x}(0, s)$ gives an extra equation yielding the following system of equations

$$
\left(\begin{array}{cc}
J_{0}^{a} & Y_{0}^{a} \\
J_{1}^{a} & Y_{1}^{a}
\end{array}\right)\left(\begin{array}{c}
A \\
B
\end{array}\right)=\left(\begin{array}{c}
1 \\
\imath s \sqrt{\frac{a}{g}}
\end{array}\right) \hat{y}(s)
$$

where $\mathcal{C}^{a}=\mathcal{C}\left(2 \imath s \sqrt{\frac{a}{g}}\right)$, for $\mathcal{C}=J_{0}, J_{1}, Y_{0}, Y_{1}$. The solutions of this system are

$$
\begin{aligned}
A & =\left(-\imath \pi s \sqrt{\frac{a}{g}} Y_{1}^{a}-\pi s^{2} \frac{a}{g} Y_{0}^{a}\right) \hat{y}(s) \\
B & =\left(\imath \pi s \sqrt{\frac{a}{g}} J_{1}^{a}+\pi s^{2} \frac{a}{g} J_{0}^{a}\right) \hat{y}(s)
\end{aligned}
$$

Similarly, noting $\mathcal{C}^{x}=\mathcal{C}\left(2 \imath s \sqrt{\frac{x+a}{g}}\right)$, for $\mathcal{C}=$
$J_{0}, J_{1}, Y_{0}, Y_{1}$ we get

$$
\begin{aligned}
& \hat{X}(x, s)= \\
& \pi s^{2} \frac{a}{g}\left(J_{0}^{a} Y_{0}^{x}-Y_{0}^{a} J_{0}^{x}\right) \hat{y}(s)+\imath \pi s \sqrt{\frac{a}{g}}\left(J_{1}^{a} Y_{0}^{x}-Y_{1}^{a} J_{0}^{x}\right) \hat{y}(s)
\end{aligned}
$$

In order to turn this equation back into the time domain, we look for integral representation of the operators $\left(J_{0}^{a} Y_{0}^{x}-Y_{0}^{a} J_{0}^{x}\right)$ and $\left(J_{1}^{a} Y_{0}^{x}-Y_{1}^{a} J_{0}^{x}\right)$. We use the following classical integral representations, derived from [1]

$$
\begin{aligned}
J_{0}(z) & =\frac{1}{\pi} \int_{0}^{\pi} \exp (\imath z \cos \theta) d \theta \\
Y_{0}(z) & =\frac{2}{\pi^{2}} \int_{0}^{\pi} \exp (\imath z \cos \theta)\left(\gamma+\ln \left(2 z \sin ^{2} \theta\right)\right) d \theta \\
J_{1}(z) & =\frac{1}{\imath \pi} \int_{0}^{\pi} \exp (\imath z \cos \theta) \cos \theta d \theta \\
Y_{1}(z) & =\frac{2}{\imath \pi^{2}} \int_{0}^{\pi} \exp (\imath z \cos \theta) \ldots \\
& \left(\gamma \cos \theta+\ln \left(2 z \sin ^{2} \theta\right) \cos \theta-\frac{\imath}{z}\right) d \theta
\end{aligned}
$$

where $\gamma$ is the Euler-Mascheroni constant. These allows us to derive the following relations

$$
\begin{aligned}
& J_{0}(x) Y_{0}(y)-J_{0}(y) Y_{0}(x)= \\
& \frac{2}{\pi^{3}} \iint_{[0, \pi]^{2}} \exp (\imath x \cos \theta+\imath y \cos \phi) \ln (y / x) d \theta d \phi \\
& J_{1}(x) Y_{0}(y)-Y_{1}(x) J_{0}(y)= \\
& \frac{2}{\imath \pi^{3}} \iint_{[0, \pi]^{2}} \exp (\imath x \cos \theta+\imath y \cos \phi) \ldots \\
& \left(\ln \left(\frac{y \sin ^{2} \phi}{x \sin ^{2} \theta}\right) \cos \theta+\frac{i}{x}\right) d \theta d \phi
\end{aligned}
$$


When substituted in (12) these relations give

$\hat{X}(x, s)=$

$\left(\frac{1}{\pi^{2}} \iint_{[0, \pi]^{2}} \exp (-\delta(x, \theta, \phi) s) d \theta d \phi\right.$

$+s \frac{2}{\pi^{2}} \sqrt{\frac{a}{g}} \iint_{[0, \pi]^{2}} G(x, \theta, \phi) \cos \theta \exp (-\delta(x, \theta, \phi) s) d \theta d \phi$

$\left.+s^{2} \frac{2 a}{\pi^{2} g} \iint_{[0, \pi]^{2}} G(x, \theta, \phi) \exp (-\delta(x, \theta, \phi) s) d \theta d \phi\right) \hat{y}(s)$

where $G(x, \theta, \phi)=\ln \left(\frac{\sqrt{x+a} \sin ^{2} \phi}{\sqrt{a} \sin ^{2} \theta}\right)$ and $\delta(x, \theta, \phi)=$ $\frac{2}{\sqrt{g}}(\sqrt{a} \cos \theta+\sqrt{x+a} \cos \phi)$.

A remarkable fact is that these expressions involve only entire functions of $s$. Indeed one can clearly recognize distributed delay operators with $G$ and $\delta$ as the gain and delay distributions. Turning this relation back into the time domain gives

$$
\begin{aligned}
& X(x, t)= \\
& \frac{1}{\pi^{2}} \iint_{[0, \pi]^{2}} y(t-\delta(x, \theta, \phi)) d \theta d \phi \\
& +\frac{2}{\pi^{2}} \sqrt{\frac{a}{g}} \iint_{[0, \pi]^{2}} G(x, \theta, \phi) \cos \theta \dot{y}(t-\delta(x, \theta, \phi)) d \theta d \phi \\
& +\frac{2 a}{\pi^{2} g} \iint_{[0, \pi]^{2}} G(x, \theta, \phi) \ddot{y}(t-\delta(x, \theta, \phi)) d \theta d \phi
\end{aligned}
$$

This is how the state of the system writes in terms of the flat output $y$ and its first two time derivatives using distributed delays.

\section{Conclusion}

We have shown that, around the stable vertical position, heavy chain systems with or without load, with constant or variable section are "flat": the trajectories of these systems are parameterizable by the trajectories of their free ends. Relations (5), (8), (10) show that such parameterizations involve operators of compact supports.

It is surprising that such parameterizations can also be applied around the inverse and unstable vertical position. For the homogeneous heavy chain, we just have to replace $g$ by $-g$ to obtain a family of smooth solutions to the elliptic equation (singular at $x=0$ )

$$
\frac{\partial}{\partial x}\left(g x \frac{\partial X}{\partial x}\right)+\frac{\partial^{2} X}{\partial t^{2}}=0
$$

by the following integral

$$
X(x, t)=\frac{1}{2 \pi} \int_{-\pi}^{\pi} y(t+2 \imath \sqrt{x / g} \sin \theta) d \theta,
$$

where $y$ is now an holomorphic function in $\mathbb{R} \times$ $[-2 \sqrt{L / g},+2 \sqrt{L / g}]$ that is real on the real axis. This parameterization can still be used to solve the motion planning problem in spite of the fact that the Cauchy problem associated to this elliptic equation is not wellposed in the sense of Hadamard.

Acknowledgement The authors would like to thank Michel Fliess and Philippe Martin for fruitful discussion on Paley-Wiener theorem and series expansion and Olivier Crevoiserat for his work on this subject.

\section{References}

[1] M. Abramowitz and I.A. Stegun. Handbook of Mathematical Functions. Dover, New York, 1965.

[2] F. Boustany. Commande non linéaire adaptative de systèmes mécaniques de type pont roulant, stabilisation frontière d'EDP. PhD thesis, École des Mines de Paris, 1996.

[3] M. Fliess, J. Lévine, Ph. Martin, and P. Rouchon. Flatness and defect of nonlinear systems: introductory theory and examples. Int. J. Control, 61(6):1327-1361, 1995.

[4] M. Fliess, J. Lévine, Ph. Martin, and P. Rouchon. A Lie-Bäcklund approach to equivalence and flatness of nonlinear systems. IEEE Trans. Automat. Control, 44:922-937, 1999.

[5] M. Fliess, Ph. Martin, N. Petit, and P. Rouchon. Active signal restoration for the telegraph equation. In CDC 99, Phenix, december 1999.

[6] S. Hansen and E. Zuazua. Exact controllability and stabilization of a vibrating string with an interior point mass. SIAM J. Contr. Opt., 33:1357-1391, 1995.

[7] J. L. Lions. Contrôlabilité Exacte, Perturbations et Stabilisation de Systèmes Distribués, volume 1. Masson, Paris, 1988.

[8] J. L. Lions. Exact controllability, stabilization and perturbations for distributed systems. SIAM Rev., 30:1-68, 1988.

[9] Ph. Martin and P. Rouchon. Flatness and sampling control of induction motors. In Proc. IFAC World Congress, pages 389-394, San Francisco, 1996.

[10] R. M. Murray. Trajectory generation for a towed cable flight control system. In Proc. IFAC World Congress, pages 395-400, San Francisco, 1996.

[11] N. Petit and P. Rouchon. Flatness of heavy chain systems. SIAM J. Control Optimization, 40:475-495, 2001. 